\title{
EFFECTUATION: UMA REVISÃO SISTEMÁTICA DA LITERATURA
}

Nayara Cristina Mendonça Zanata ${ }^{1}$

Carolina Camargo Felice ${ }^{1}$

Camila Maria De Oliveira $^{1}$

Márcia Freire De Oliveira ${ }^{1}$

${ }^{1}$ Faculdade de Gestão e Negócios - Universidade Federal de Uberlândia 


\section{EFFECTUATION: UMA REVISÃO SISTEMÁTICA DA LITERATURA}

\section{Resumo:}

O presente trabalho tem por objetivo avaliar, por meio da revisão sistemática, a produção bibliográfica sobre effectuation da base de dados Periódicos Capes publicada entre 2012 e 2018. Diante dos 47 artigos selecionados, foi possível agrupar os estudos nos seguintes temas: (A) O processo decisório e a lógica do causation e effectuation; B) $\mathrm{O}$ causation e effectuation no processo de desenvolvimento de novos produtos/empresas, tecnologia e inovação; C) $\mathrm{O}$ causation e effectuation na aprendizagem do processo empreendedor, (D) Revisões de estudos acadêmicos sobre effectuation. Os resultados também apontam um crescente aumento do número de artigos sobre effectuation ao longo do período pesquisado e uma predominância de artigos publicados em periódicos internacionais.

Palavras-chave: Effectuation. Causation. Revisão Sistemática. 


\section{Introdução}

O termo effectuation, designado pela autora Saras Saravasthy (2008), refere-se a uma lógica geral de ação, ensinável e aprendível, de caráter intrinsecamente criativo, que procura a partir dos meios disponíveis, desenvolver objetivos, que podem ser reformulados. A lógica efectual originou-se da tese de doutorado de Saravasthy, por meio da realização de uma pesquisa com 45 empreendedores (SARAVASTHY, 2008; SILVERZAHN, 2014).

Deste modo, a partir do modo como os empreendedores solucionavam problemas ou situações correspondentes ao empreendedorismo, Sarasvathy (2008) concluiu a existência de cinco princípios do effectuation, os quais os empreendedores podem ou não ter ciência, sendo eles: (1) pássaro na mão: começar a agir com o que se tem disponível; (2) perda aceitável, ao invés de retorno esperado; (3) colcha de retalhos: construir parcerias ao invés de se preocupar com os concorrentes ; (4) fazer do limão uma limonada: tirar proveito das contingências e adversidades e; (5) piloto do avião: controlar um futuro imprevisível ao invés de tentar prevê-lo (SARAVASTHY, 2008; SILVERZAHN, 2014).

A lógica do effectuation é um modelo alternativo à lógica clássica, tradicional de tomada de decisão (causal) (PELOGIO et. al., 2013). A lógica causal, possui caráter determinista, pois busca a otimização ao focar em um planejamento para encontrar um caminho mais eficaz em prol do objetivo pré-estabelecido, dando ênfase à predição. Já, a lógica efectual não determinista, utiliza da lógica da criação para que, a partir dos meios que apresentam, surjam possíveis efeitos (SILVERZAHN, 2014; SARAVASTHY, 2008).

No que confere às lógicas efectual e causal, Saravasthy (2008) salienta que as mesmas podem ser usadas por uma mesma pessoa, dependendo das cirscunstâncias bem como do nível de expertise do empreendedor e em qual estágio de vida a empresa se encontra. (SARASVATHY, 2008).

Os estudos sobre effectuation vêm ganhando relevância ao longo dos anos. A pesquisa de Matalamäki (2017) apontou que em âmbito internacional de 1998 até 2010 as publicações anuais em média eram de 2 artigos, chegando a 12 artigos em 2015 e 16 artigos em 2017. Portanto percebe-se que, ao menos internacionalmente, houve um aumento significativo no número de artigos sobre a temática.

Dentre desse contexto, e considerando também as publicações nacionais, a presente pesquisa pretende responder a seguinte questão de pesquisa: qual é a produção bibliográfica sobre effectuation publicada em periódicos nacionais e internacionais de 2012 a 2018 ?

Deste modo, o estudo em pauta tem por objetivo avaliar, por meio de revisão sistemática, a produção bibliográfica de artigos nacionais e internacionais sobre effectuation da base de dados Periódicos Capes publicada entre 2012 e 2018.

A revisão sistemática é um método apoiado na revisão da literatura que a partir de uma questão de pesquisa estabelecida de forma clara é feita a identificação, seleção e avaliação dos artigos, buscando sintetizar evidências importantes, envolvendo a aplicação de critérios explícitos e procedimentos padronizados (FUCHS; PAIM, 2010). Conforme Tranfield, Denyer e Smart (2013) a revisão sistemática, normalmente utilizada em pesquisas da área de saúde, pode contribuir com os estudos de gestão, conferindo-lhes melhor rigor metodológico.

Os métodos para elaboração de revisões sistemáticas preveem: (1) elaboração da pergunta de pesquisa; (2) busca na literatura; (3) seleção dos artigos; (4) extração dos dados;
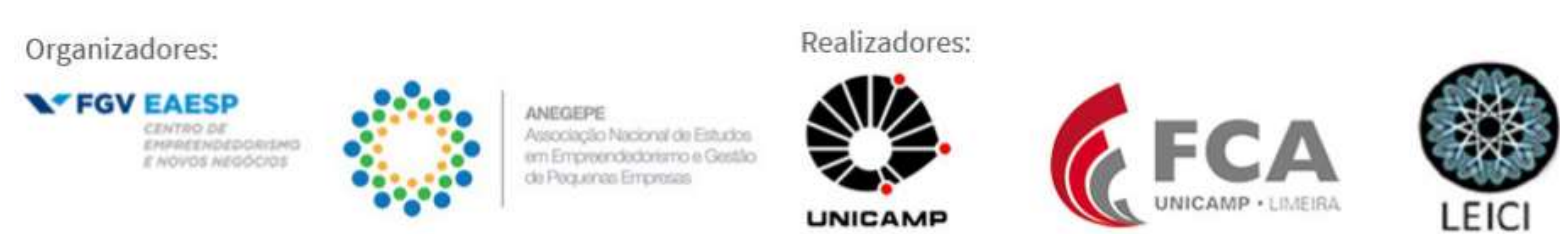
(5) avaliação da qualidade metodológica; (6) síntese dos dados; (7) avaliação da qualidade das evidências; e (8) redação e publicação dos resultados (GALVÃO; PEREIRA, 2014).

$\mathrm{O}$ presente artigo, além desta introdução está dividido em 3 seções. Na primeira, o método usado para a realização da pesquisa é detalhado; na segunda, os resultados são apresentados e desdobrados posteriormente nas seguintes subseções: o processo decisório e a lógica do causation e effectuation; o causation e effectuation no processo de desenvolvimento de novos produtos/empresas, tecnologia e inovação; o causation e effectuation na aprendizagem do processo empreendedor e; Revisão de estudos acadêmicos relacionados à effectuation. Por fim, as considerações finais são descritas na quarta seção.

\section{Método}

A revisão sistemática da presente pesquisa foi feita conforme as seguintes etapas: (1) a escolha do tema (effectuation), elaboração da questão de pesquisa, escolha da base de dados a ser utilizada e determinação dos termos de busca dos artigos (2) identificação e localização, ocasionada pela busca direta, no acervo eletrônico do Portal do Periódicos da CAPES; (4) compilação dos artigos encontrados; (5) fichamento: ordenação dos artigos por meio de fichas constando: nome do artigo; autores; periódico; ano de publicação; objetivos; método e principais resultados; (6) análise e interpretação, do material bibliográfico encontrado e; por fim;(7) redação dos resultados encontrados.

A pesquisa dos artigos foi realizada mediante a busca eletrônica nas bases de dados no Portal de Periódicos CAPES. Foram utilizados os seguintes campos para a realização da busca busca: I) palavra effectuation apenas no título; II) ano de publicação - artigos publicados entre 2012 e 2018 e; III) tipo de material restrito a artigos.

A partir da busca supracitada, foram encontrados 60 artigos que, diante de uma leitura seletiva dos resumos, foram preferidos 47. Os critérios de exclusão foram: I) artigos que não se apresentavam em línguas inglesa ou portuguesa e II) artigos que não abordavam o tema effectuation. Deste modo, o corpus que representou o material de análise foi constituído por 47 artigos. Foi feita uma segunda leitura completa dos artigos tabulando-os nas seguintes dimensões: nome do artigo; autores; periódico; ano de publicação; objetivos; método e principais resultados.

Desta forma, pode-se obter uma análise dos estudos apurados, viabilizando um cenário geral da produção científica internacional e nacional relacionado a effectuation. Após a tabulação, levantaram-se os temas que emergiram da leitura e análise dos artigos, agrupandoos de acordo com a semelhança entre os estudos.

\section{Resultados}

Dos 47 artigos constituintes do corpus de análise 8 foram publicados em periódicos nacionais e 39 em periódicos internacionais, o que mostra uma baixa expressividade de artigos sobre Effectuation nas revistas brasileiras. Em termos da quantidade de periódicos encontrouse 39, sendo 34 internacionais e 5 nacionais, observando-se uma grande variedade de revistas que publicaram sobre o tema. Grande parte dos periódicos (trinta e três), publicaram apenas um artigo relacionado ao tema. As exceções foram o Journal of Business Research e a, Revista Eletrônica de Negócios Internacionais (Intertext) com três publicações cada, e os periódicos Journal of Enterprising Culture, Journal of Small Business and Enterprise Development,

\section{Organizadores:}

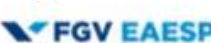

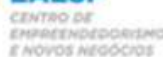

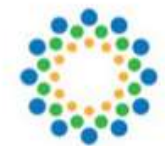

ANEOEPE

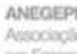

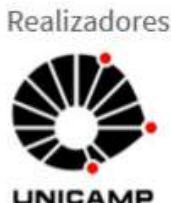

UNICAMP
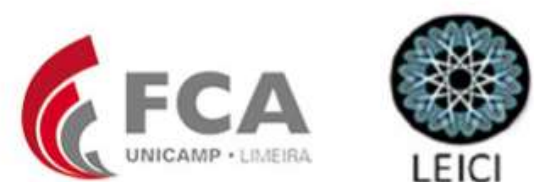
International Entrepreneurship and Management Journal, Revista de Administração Contemporânea, com duas publicações cada.

Em relação ao ano de publicação dos artigos, um artigo foi publicado em 2012; três em 2013; cinco em 2014; dez em 2015; oito em 2016; dezessete em 2017 e até o momento da realização desta pesquisa, 3 artigos no ano de 2018. Observa-se, portanto, uma concentração de publicações entre os anos de 2014 e 2017, revelando que os estudos referentes ao tema causation e effectuation cresceram expressivamente nestes últimos anos.

Em se tratando do número de autores por publicação, dois artigos foram publicados por um único autor cada; quinze artigos por dois autores cada; vinte artigos por três autores cada; sete por quatro autores cada; dois por cinco autores cada; e um artigo por seis autores.

Os artigos pesquisados foram agrupados segundo os seguintes temas: A) O processo decisório e a lógica do causation e effectuation; B) $\mathrm{O}$ causation e effectuation no processo de desenvolvimento de novos produtos/empresas, tecnologia e inovação; C) O causation $e$ effectuation na aprendizagem do processo empreendedor; e D) Revisão de estudos acadêmicos sobre effectuation. O quadro 1, mostra a relação dos autores com os temas.

Quadro 1: Relação de autores e temas

\begin{tabular}{|c|c|c|c|c|}
\hline 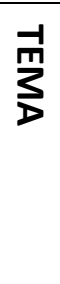 & $\begin{array}{l}\text { O processo decisório e a } \\
\text { lógica do causation } \\
\text { effectuation }\end{array}$ & $\begin{array}{lr}\text { O causation } & e \\
\text { effectuation } & \text { no } \\
\text { processo } & \text { de } \\
\text { desenvolvimento de } & \text { de } \\
\text { novos produtos } \\
\text { (empresas), tecnologia } \\
\text { e inovação }\end{array}$ & $\begin{array}{lr}\text { O causation } & e \\
\text { effectuation } & \text { na } \\
\text { aprendizagem } & \text { do } \\
\text { processo empreendedor }\end{array}$ & $\begin{array}{l}\text { Revisão de } \\
\text { estudos } \\
\text { acadêmicos } \\
\text { sobre } \\
\text { effectuation }\end{array}$ \\
\hline 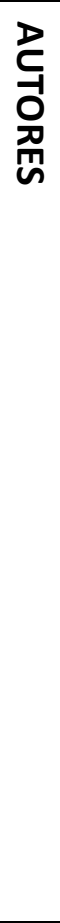 & $\begin{array}{l}\text { Chetty, Ojala e Leppäaho } \\
\text { (2015); Parida et. al. (2016); } \\
\text { Weltera e Kimb (2017); } \\
\text { Galkinaa e Lundgren- } \\
\text { Henrikssonb (2017); Galkina e } \\
\text { Chetty (2015); Futterer, } \\
\text { Schmidt e Heidenreich (2018); } \\
\text { Sitoh, Pan e Yu (2014); Porto e } \\
\text { Mello (2015); Matalamäki et. } \\
\text { al. (2017); Pelogio et. al. } \\
\text { (2013);Matta e Mello (2014); } \\
\text { Evald e Senderovitz (2013); } \\
\text { Long, Xia e Hu (2017); La } \\
\text { Cruz, Jover e Gras (2017), } \\
\text { Schmidt, Bendig e Brettel } \\
\text { (2017); Eyana, Masurel e Paas } \\
\text { (2017); Laine e Galkina } \\
\text { (2016); Sarmento, Carvalho e } \\
\text { Dib (2016); Reymen et. al. } \\
\text { (2015); Yua et. al. (2017); } \\
\text { Reymen et. al. (2016); Leucz e } \\
\text { Andreassi (2015); Hes et. al. } \\
\text { (2017). }\end{array}$ & $\begin{array}{l}\text { Berends et. al. (2014); } \\
\text { Villania, Lindera e } \\
\text { Grimaldib (2017); } \\
\text { Ortega, García e Santos } \\
\text { (2017); Daniel, Di } \\
\text { Domenico e Sharma } \\
\text { (2015); Deligianni, } \\
\text { Voudouris e Lioukas } \\
\text { (2017); } \\
\text { Roach, Ryman e } \\
\text { Makani (2016); Jisr e } \\
\text { Maamari (2017); } \\
\text { Guo,Cai e Zhang } \\
\text { (2016); Cai et. al. } \\
\text { (2017) }\end{array}$ & $\begin{array}{l}\text { Stroea, Paridab e } \\
\text { Wincentb (2018); } \\
\text { Murdock e Varnes } \\
\text { (2018); Faia, Rosa e } \\
\text { Machado (2014); } \\
\text { Helmersson e Mattsson } \\
\text { (2012); Salusse e } \\
\text { Andreassi (2016); } \\
\text { Melinda, Sutanto e } \\
\text { Christian (2015), } \\
\text { Agogué, Lundqvist e } \\
\text { Middleton (2015); } \\
\text { Günzel-Jensen (2017); } \\
\text { Robinson en } \\
\text { Laskovaia, Shirokova e } \\
\text { Morris (2017); Ahn et. al. } \\
\text { (2015); Gut } \\
\text { Dutta, Gwebu e Wang } \\
\text { (2013); Urban e } \\
\text { Heydenrych (2015), } \\
\text { Melinda, Sutanto e } \\
\text { Christian (2014). }\end{array}$ & $\begin{array}{l}\text { Matalamäki } \\
\text { (2017); Pelogio } \\
\text { e Rocha (2016) }\end{array}$ \\
\hline
\end{tabular}

Fonte: Elaboração própria 
Analisando o quadro 1 percebe-se que o tema $\mathrm{D}$ foi o que apresentou o menor número de artigos, apenas dois. Já o tema $\mathrm{A}$ foi o que apresentou o maior número de artigos, vinte e três, e o tema B e C apresentou, respectivamente, nove e treze artigos. Nas quatro subseções seguintes, serão detalhados os estudos relacionados a cada tema.

\section{O processo decisório e a lógica do causation e effectuation}

A maior parte dos estudos relacionados ao tema em questão, propuseram-se a compreender as abordagens causation e rffectuation como fatores determinantes no processo decisório dos empreendedores (PARIDA et. al., 2016; GALKINAA; LUNDGRENHENRIKSSONB, 2017; STOH; PAN; YU, 2014; PELOGIO et. al., 2013; SCHMIDT; BENDIG; BRETTEL, 2017; REYMEM et. al, 2015) principalmente no que tange ao desenvolvimento estratégico e a exploração de novos mercados, por meio da internacionalização (CHETTY; OJALA; LEPPÄAHO, 2015; PORTO; MELLO, 2015; MATTA; MELLO, 2014; SARMENTO; CARVALHO; DIB, 2016). Outros estudos buscaram analisar como estas duas abordagens influenciam o comportamento do empreendedor em meio de contextos incertos e arriscados (WELTERA; KIMB, 2017; GALKINA; CHETTY, 2015; FUTTERER; SCHMIDT; HEIDENREICH, 2018; EYANA; MASUREL; PAAS, 2017; LAINE; GALKINA, 2016;).

Além disso, alguns estudos procuraram investigar o desenvolvimento (e crescimento) de negócios, considerando a teoria causation e effectuation como base para compreender os fatores determinantes de sucesso para estes empresários (MATALAMÄKI et. al, 2017; EVALDO; SENDEROVITZ, 2013; EYANNA; MASUREL; PAAS, 2017; YUA et. al., 2017; REYMEM et.al., 2016; LEUCZ; ANDREASSI, 2015). Já os autores Long, Xia e Hu (2017) buscaram, em seus estudos, construir um modelo de pesquisa a partir das perspectivas de effectuation e oportunidade empresarial. Por fim, o artigo de Sulaiman et. al. (2017) procurou compreender a relação até então não considerada entre o capital social e os conceitos relacionados à effectuation.

Os resultados dos estudos apontaram que os empreendedores tendem a entrelaçar as lógicas da effectuation e causation para realizar a tomada de decisão (CHETTY; OJALA. LEPPÄAHO, 2015; GALKINAA; LUNDGREM-HENRIKSSONB, 2017; MATALAMÄKI et. al., 2017; REYMEN et. al., 2015), entretanto a effectuation é vista por alguns autores como abordagem dominante (MATALAM ̈̈KI et. al., 2017; EVALDO; SENDEROVITZ, 2013; SCHIMIDT; BENDIG; BRETTEL, 2017; LAINE; GALKINA, 2016; REYMEM et. al., 2016). Além disso, os autores Sitoh, Pan e Yu (2014), descobriram que existem quatro configurações de tomada de decisão: centrada na effectuation, centrada na descoberta, centrada na causation e centrada na tática. Laina e Galkina (2016), apontaram que existem dois tipos de effectuation: a movida a oportunidades e a de sobrevivência. Essas descobertas contribuíram para a pesquisa realizada por Pelogio et. al. (2013) que apontou ser necessário usar outras abordagens que compreendam a ação do empreendedor sob uma visão não determinística e não-linear.

Long, Xia e Hu (2017) apontaram que a descoberta de oportunidades torna-se mais positiva quando os empreendedores usam a lógica da effectuation. Para selecionar e adentrar em mercados estrangeiros, os empreendedores tendem a usar Effectuation (CHETTY; OJALA. LEPPÄAHO, 2015; GALKINA; CHETTY, 2015; PORTO; MELLO, 2015), e conforme Matta e Mello (2014) no mercado internacional, normalmente, o caminho trilhado não segue o

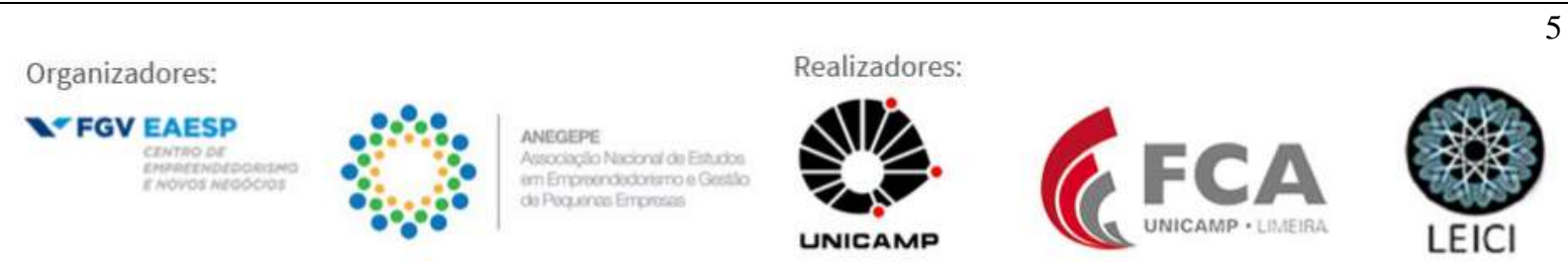


planejamento inicial. Em contextos arriscados e incertos, os estudos apontaram que a effectuation supera a causation, dando aos empresários mais precisão na previsão de cenários futuros (WELTERRA; KIMB, 2017; YUA et. al., 2017). Quanto ao desenvolvimento (e crescimento) de negócios, os estudos apontaram que a effectuation (causation) se torna mais efetiva nas configurações de alto (baixo) crescimento da indústria (FUTTERER; SCHMIDT; HEIDENREICH, 2018), porém Eyana, Masurel e Paas (2017), não encontraram suporte empírico que sustentem a hipótese que a effectuation seja superior ao causation em em firmas com desempenhos superiores. O Quadro 2 ilustra a análise geral descrita nos parágrafos anteriores.

Quadro 2: Análise geral dos artigos relacionados ao tema $\mathrm{A}$

\begin{tabular}{|c|c|c|c|c|c|c|}
\hline Autores & 1 & 2 & 3 & 4 & 5 & 6 \\
\hline Parida et. al. (2016) & $\mathrm{x}$ & & & & & \\
\hline Galkinaa e Lundgren-Henrikssonb (2017) & $\mathrm{x}$ & & & & & \\
\hline Stoh, Pan e Yu (2014) & $\mathrm{x}$ & & & & & \\
\hline Pelogio et. al. (2013) & $\mathrm{x}$ & & & & & \\
\hline Schmidt, Bendig e Brettel (2017) & $\mathrm{x}$ & & & & & \\
\hline Reymem et. al (2015) & $\mathrm{x}$ & & & & & \\
\hline Chetty, Ojala e Leppäaho (2015) & & $\mathrm{X}$ & & & & \\
\hline Porto e Mello (2015) & & $\mathrm{X}$ & & & & \\
\hline Matta e Mello (2014) & & $\mathrm{X}$ & & & & \\
\hline Sarmento, Carvalho e Dib (2016) & & $\mathrm{X}$ & & & & \\
\hline Weltera e Kimb (2017) & & & $\mathrm{x}$ & & & \\
\hline Galkina e Chetty (2015) & & & $\mathrm{X}$ & & & \\
\hline Futterer, Schmidt e Heidenreich (2018) & & & $\mathrm{x}$ & & & \\
\hline Eyana, Masurel e Paas (2017) & & & $\mathrm{x}$ & $\mathrm{x}$ & & \\
\hline Laine e Galkina (2016) & & & $\mathrm{x}$ & & & \\
\hline Matalamäki et. al (2017) & & & & $\mathrm{x}$ & & \\
\hline Evaldo e Senderovitz (2013) & & & & $\mathrm{x}$ & & \\
\hline Leucz; Andreassi (2015) & & & & $\mathrm{x}$ & & \\
\hline Yua et. al. (2017) & & & & $\mathrm{x}$ & & \\
\hline Reymem et.al. (2016) & & & & $\mathrm{x}$ & & \\
\hline Long, Xia e Hu (2017) & & & & & $\mathrm{x}$ & \\
\hline Sulaiman et. al. (2017) & & & & & & $\mathrm{x}$ \\
\hline \multicolumn{7}{|c|}{$\begin{array}{l}\text { 1) A influência do effectuation no processo decisório; } \\
\text { 2) A influência do effectuation no processo decisório no que tange a decisões estratégicas; } \\
\text { 3) Comportamento do empreendedor em meio a contextos arriscados e incertos; } \\
\text { 4) Fatores determinantes de sucesso em empresários; } \\
\text { 5) Elaboração de um modelo de pesquisa; } \\
\text { 6) Relação entre capital social e effectuation. }\end{array}$} \\
\hline
\end{tabular}
Fonte: Elaboração própria

\section{O causation e effectuation no processo de desenvolvimento de novos produtos (empresas),} tecnologia e inovação

Dentre os estudos utilizados para este artigo, alguns se destacaram por trazer uma discussão acerca do desenvolvimento de novos produtos (empresas), tecnologia e inovação. Estes artigos procuraram investigar como o processo de inovação e desenvolvimento de novos 
produtos estabelece a lógica do effectuation (BERENDS et. al., 2014; ORTEGA; GARCIA; SANTOS, 2017) bem como, identificar as características no lançamento de empresas bem sucedidas (VILLANIA; LINDERA; GRIMALDIB, 2017). Ainda em relação à inovação, o estudo realizado por Roach, Ryman e Makani (2016) propôs uma escala que explicasse a construção de effectuation neste contexto. Também foi possível identificar estudos que se propuseram ampliar o conhecimento acerca do "homeoffice", onde buscaram trazer as contribuições da effectuation para o contexto em questão (DANIEL; DI DOMENICO; SHARMA, 2015).

Já os estudos de Deligianni, Voudouris e Lioukas (2017) e de Cai et. al. (2017), tinham como foco a effectuation e a relação entre diversificação e desempenho em empreendimentos novos. Outros estudos buscaram compreender como o conhecimento tácito afeta o desempenho da inovação, contribuindo para o effectuation (JISR; MAAMARI, 2017). Por fim, Guo, Cai e Zhang (2016) testaram um modelo teórico que vincula os princípios do effectuation ou causation ao crescimento de novos empreendimentos na internet através do agrupamento de recursos (pioneirismo ou estabilização).

Os resultados indicaram que na inovação e desenvolvimento de produtos a lógica da effectuation inicial se torna causation ao longo do tempo (BERENDS et. al., 2014), entretanto outros estudos demonstraram a presença de um comportamento híbrido com predominância para a effectuation em projetos com um maior grau de inovação e incerteza (ORTEGA; GARCÍA; SANTOS, 2017). Villania, Lindera e Grimaldib (2017) identificaram diferenças de abordagens em startups BC (baseada em ciência), onde existe mais confiança no planejamento, e em startups nBC (não baseada em ciência), que prevalece a flexibilidade. Já os estudos referente aos "homeoffice" indicaram que a effectuation pode ser associada à baixos níveis de auto eficácia e de experiência empresarial, uma vez que esses contextos de negócios oferecem baixa perda acessível e oportunidades para experiências rápidas (DANIEL; DI DOMENICO; SHARMA, 2015). Quanto os resultados que direcionam a diversificação-desempenho de novos negócios, os processos de effectuation mostraram exercer um efeito positivo (DELIGIANNI; VOUDOURIS; LIOUKAS, 2017; JISR; MAAMARI, 2017; CAI et. al., 2017). Roach, Ryman e Makani (2016), trazem como resultados de seus estudos uma escala mais cautelosa para se medir a inovação, cujas medidas foram divididas em orientação para inovação e a inovação de produtos/serviços. Por fim, Guo, Cai e Zhang (2016) associam positivamente a effectuation e o Causation ao crescimento de novos empreendimentos na internet.

O Quadro 3 ilustra a análise geral descrita nos parágrafos anteriores.

\section{Quadro 3: Análise geral dos artigos relacionados ao tema B}

\begin{tabular}{|l|l|l|l|l|l|l|l|}
\hline Autores & 1 & 2 & 3 & 4 & 5 & 6 & 7 \\
\hline Berends et. al. (2014) & $\mathrm{x}$ & & & & & & \\
\hline Ortega, Garcia e Santos (2017) & $\mathrm{x}$ & & & & & & \\
\hline Villania, Lindera e Grimaldib (2017) & & $\mathrm{x}$ & & & & & \\
\hline Roach, Ryman e Makani (2016) & & & $\mathrm{x}$ & & & & \\
\hline Daniel, Di Domenico e Sharma (2015) & & & & $\mathrm{x}$ & & & \\
\hline Deligianni, Voudouris e Lioukas (2017) & & & & & $\mathrm{x}$ & & \\
\hline Cai et. al. (2017) & & & & & & $\mathrm{x}$ & \\
\hline Jisr e Maamari (2017) & & & & & & & \\
\hline Guo, Cai e Zhang (2016) & & & \\
\hline
\end{tabular}

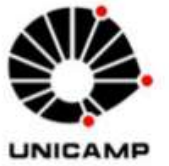


1) Como o processo de inovação e desenvolvimento de novos produtos estabelece a lógica do Effectuation;

2) Características no lançamento de empresas bem sucedidas;

3) Construção de uma escala que explicasse a construção de effectuation;

4) O "homeoffice" e as contribuições da effectuation;

5) A effectuation e a relação entre diversificação e desempenho em empreendimentos novos;

6) Como o conhecimento tácito afeta o desempenho da inovação;

7) Testar um modelo teórico.

Fonte: Elaboração própria

\section{O causation e effectuation na aprendizagem do processo empreendedor}

$\mathrm{O}$ causation e effectuation tornam-se bastante relevantes quando analisamos a capacidade de aprendizagem do processo empreendedor, uma vez que as características predominantes em pessoas empreendedoras podem estar relacionadas com o nascimento ou com o domínio ao longo do tempo. A partir deste princípio, vários acadêmicos passaram a produzir estudos relacionados ao tema em questão. Alguns estudos buscaram compreender como a subjetividade ("a paixão empreendedora") conduzem a uma lógica de tomada de decisão causation ou effectuation (STROEA; PARIDAB; WINCENTB, 2018). Já outros estudos buscaram descrever, expandindo além da effectuation, como o empreendedor é continuamente transformado (MURDOCK; VARNES, 2018). Faria et. al. (2014), buscou apresentar um estudo mais técnico com o intuito de relacionar o grau de alerta empreendedor com as abordagens causation e effectuation.

A orientação comportamental dos empreendedores em relação ao effectuation e causation foi estudada por Helmersson e Mattsson (2012). Alguns estudos se destacaram por compreender, categorizar e analisar a eficácia de metodologias de ensino de empreendedorismo em função da teoria effectuation (SALUSSE; ANDREASSI, 2016; MELINDA; SUTANTO; CRISTIAN, 2015; GÜNZEL-JENSEN; ROBINSON, 2017; AHN et. al., 2015; MERLINDA; SUTANTO; CHRISTIAN, 2014). Já os autores Agogué, Lundqvist e Middleton (2015) buscaram entender o desvio mental nas tomadas de decisão em empreendimentos tecnológicos, usando como base de análise o causation e o effectuation. De forma parecida, outros estudos, analisaram os processos cognitivos e sociais na explicação do desempenho do empreendimento em diferentes contextos culturais (LASKOVAIA; SHIROKOVA; MORRIS, 2017; LA CRUZ, JOVER, GRAS, 2017). Por fim, alguns autores buscaram relacionar o effectuation à orientação tecnológica e desempenho empresarial, destacando o conhecimento e experiência do empreendedor (DUTTA; GWEBU; WANG, 2013; URBAN E HEYDENRYCH, 2015).

Os resultados apontaram que mais do que paixão, a auto eficácia empresarial e a percepção de risco em conjunto é que leva ao uso de uma lógica causation e effectuation. Desta forma, diferentes fatores de nível individual interagem para influenciar a escolha do empreendedor para a lógica de decisão (STROEA; PARIDAB; WINCENTB, 2018). Também foi encontrado uma relação positiva entre alerta empreendedor $\mathrm{e}$ as abordagens empreendedoras, especialmente para o causation (FAIA et. al., 2014). Helmersson e Mattsson (2012), postularam que as orientações de effectuation e causation podem ser consideradas características genéricas dos gerentes em geral. Por fim, Laskovaia, Shirokova e Morris (2017) concluíram que o raciocínio empresarial é construído não apenas pelas características individuais dos empreendedores, mas também pelos aspectos do contexto social no qual estão inseridos.
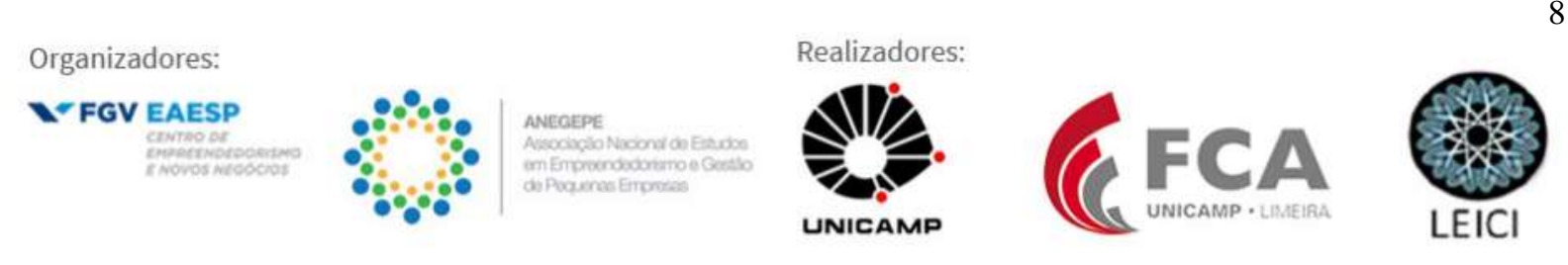
As conclusões da pesquisa realizada por Merlinda, Sutanto e Christian(2014) sugerem que o empreendedorismo é uma ciência que pode ser ensinada usando o processo de educação, desde que se utilize métodos de aprendizagem adequados, programas de treinamento e modelos de desenvolvimento do empreendedorismo. Desta forma, o modelo dinâmico de effectuation pode ser utilizado como elemento integrador (SALUSSE; ANDREASSI, 2016). Além disso, a aprendizagem baseada em effectuation indicou melhores resultados nos seguintes indicadores 1) descoberta de ideias de negócios, (2) minimização de risco, (3) flexibilidade de negócios, (4) networking e (5) utilização de recursos (MERLINDA; SUTANTO; CHRISTIAN, 2015).

O Quadro 4 ilustra a análise geral descrita nos parágrafos anteriores.

\section{Quadro 4: Análise geral dos artigos relacionados ao tema C}

\begin{tabular}{|c|c|c|c|c|c|c|c|c|}
\hline Autores & 1 & 2 & 3 & 4 & 5 & 6 & 7 & 8 \\
\hline Stroea, Paridab e Wincentb (2018) & $\mathrm{x}$ & & & & & & & \\
\hline Murdock e Varnes (2018) & & $\mathrm{x}$ & & & & & & \\
\hline Faria et. al. (2014) & & & $\mathrm{X}$ & & & & & \\
\hline Helmersson e Mattsson (2012) & & & & $\mathrm{x}$ & & & & \\
\hline Salusse e Andreassi (2016) & & & & & $\mathrm{x}$ & & & \\
\hline Melinda, Sutanto e Cristian (2015) & & & & & $\mathrm{x}$ & & & \\
\hline Günzel-Jensen e Robinson (2017) & & & & & $\mathrm{x}$ & & & \\
\hline Ahn et. al. (2015) & & & & & $\mathrm{x}$ & & & \\
\hline Merlinda, Sutanto e Christian (2014) & & & & & $\mathrm{x}$ & & & \\
\hline Agogué, Lundqvist e Middleton (2015) & & & & & & $\mathrm{x}$ & & \\
\hline Laskovaia, Shirokova e Morris (2017) & & & & & & & $\mathrm{x}$ & \\
\hline La Cruz, Jover e Gras (2017) & & & & & & & $\mathrm{x}$ & \\
\hline Dutta, Gwebu e Wang (2013) & & & & & & & & $\mathrm{x}$ \\
\hline Urban e Heydenrych (2015) & & & & & & & & $\mathrm{x}$ \\
\hline \multicolumn{9}{|c|}{$\begin{array}{l}\text { 1) Como a subjetividade ("a paixão empreendedora") conduzem a uma lógica de tomada de decisão causation ou } \\
\text { effectuation; } \\
\text { 2) Descrição de como o empreendedor é continuamente transformado; } \\
\text { 3) Relação do grau de alerta empreendedor com as abordagens causation e effectuation } \\
\text { 4) Orientação comportamental dos empreendedores; } \\
\text { 5) Compreensão, categorização e análise da eficácia de metodologias de ensino de empreendedorismo em função } \\
\text { da teoria effectuation; } \\
\text { 6) Compreensão do desvio mental nas tomadas de decisão em empreendimentos tecnológicos; } \\
\text { 7) Análise dos processos cognitivos e sociais na explicação do desempenho do empreendimento em diferentes } \\
\text { contextos culturais; } \\
\text { 8) Relação entre o effectuation e a orientação tecnológica e desempenho empresarial, destacando o conhecimento } \\
\text { e experiência do empreendedor; }\end{array}$} \\
\hline
\end{tabular}

Fonte: Elaboração própria

\section{Revisão de estudos acadêmicos sobre effectuation}

A revisão de estudos acadêmicos é de significativa importância, uma vez que possibilita uma visão geral dos estudos sobre um determinado assunto em período especifico.

Matalamäki (2017), a partir da revisão de 81 artigos de revistas acadêmicas procurou abordar os estágios de desenvolvimento da teoria da effectuation e destacar a progressão dos artigos conceituais no cenário cientifico atual. Como resultados, ele apresentou os quatros 
fluxos principais ligados à teoria da effectuation: inovação e desenvolvimento de produtos, internacionalização, effectuation e causation simultaneamente, e experiência empresarial.

Por fim, os autores Pelogio e Rocha (2016) buscaram realizar uma construção teórica destinada a expor e discutir o tema empreendedorismo e estratégia sob a ótica da lógica effectuation. Para isso, os autores realizaram uma pesquisa bibliográfica, exploratória com abordagem e análise qualitativa. Suas conclusões apontaram o modelo de decisão baseada na na lógica effectuation como modelo alternativo para a análise de criação de empresas, sendo muitas vezes mais adequado ao contexto atual.

A teoria da effectuation se mostra bastante coerente com certas realidades vivenciadas por empreendedores, que buscam se destacar em meio tão competitivo e dinâmico quanto o ambiente empresarial. Assim, evidencia-se o interesse para o estudo desta teoria em meio ao cenário científico atual.

\section{Considerações Finais}

O presente artigo utilizou o método de revisão sistemática para avaliar a produção bibliográfica sobre effectuation publicada na base de dados Periódicos Capes entre 2012 e 2018, permitindo a realização de uma pesquisa teórica com maior rigor metodológico, conforme Tranfield, Denyer e Smart (2003) e, portanto, atingindo o objetivo proposto.

Diante dos 47 artigos selecionados, foi possível agrupar os estudos nos seguintes temas: (A) O processo decisório e a lógica do causation e effectuation; B) O causation e effectuation no processo de desenvolvimento de novos produtos/empresas, tecnologia e inovação; C) $\mathrm{O}$ causation e effectuation na aprendizagem do processo empreendedor e, (D) Revisões de estudos acadêmicos sobre effectuation.

É importante destacar a baixa quantidade de estudos realizados sobre o tema em âmbito nacional, o que pode ter sido encontrado devido à disseminação tardia da teoria sobre effectuation no país, o que carece de investigação. Ao mesmo tempo, a baixa quantidade de estudos realizados no Brasil mostra que existe uma vasta possibilidade de ampliar e aprofundar os estudos sobre o tema no país. Destaca-se também o fato que grande parte dos artigos analisados levaram como tema a aprendizagem e o comportamento empreendedor, assim entende-se que os estudiosos possuem fortes crenças que o effectuation pode ser considerado uma ótima ferramenta de análise e de auxílio para os estudos em Empreendedorismo.

O empreendedorismo deve ser estudado e investigado não só para quebrar mitos, de que quem empreende assume muitos riscos, prevê o futuro ou que possuem apenas sorte (SILVERZAHN, 2014), mas para introduzir uma nova perspectiva no que diz respeito ao desempenho empresarial (SARASVATHY, 2008). Dessa forma, além de contribuir teoricamente, as pesquisas sobre effectuation podem auxiliar os empreendedores a compreender as suas possibilidades em empreender e a lógica mais adequada (causation ou effectuation) a utilizar em suas organizações. 


\section{Referências}

AGOGUÉ, M.; LUNDQVIST, M.; MIDDLETON, K. W. Mindful Deviation through Combining Causation and Effectuation: A Design Theory-Based Study of Technology Entrepreneurship. Creativity and Innovation Management. v. 24, n. 4, p. 629-644. 2015.

AHN, M. J.; YORK, A. S.; SUHARTO, Y.; DAIMN, T. On valuing biopharmaceutical product pipelines: an effectuation model and evidence. Journal of Innovation. v. 14, n. 4, p. $1-19,2015$.

BERENDS. H.; JELINEK, M.; REYMEN, I.; STULTIËNS, R. Product innovation processes in small firms: combining entrepreneurial effectuation and managerial causation. Product Development \& Management Association, v. 31, n. 3, p. 616-635. 2014.

CAI, L.; GUO, R.; FEI, Y.; LIU, Z. Effectuation, Exploratory Learning and New Venture Performance: Evidence from China. Journal of Small Business Management. v. 55, n. 3, p. 388-403. 2017.

CHETTY, S.; OJALA, A.; LEPPÄAHO, T. Effectuation and foreign market entry of entrepreneurial firms. European Journal of Marketing, v. 49, n. 9/10, p. 1436-1459. 2015.

CRUZ, M. E de la; JOVER, A. J. V.; GRAS, J. M. G. Influence of the entrepreneur's social identity on business performance through effectuation. European Research on Management and Business Economics. 2017. Disponível em : < https://doi.org/10.1016/j.iedeen.2017.11.003>. Acesso em fevereiro de 2018.

DANIEL, E. M.; DOMENICO, M. L.; SHARMA, S. Effectuation and home-based online business entrepreneurs. International Small Business Journal. v. 33. n. 8, p. 799-823. 2015.

DELIGIANNI, I.; VOUDOURIS, I.; LIOUKAS, S. Do effectuation processes shape the relationship between product diversification and performance in new ventures?

Entrepreneurship Theory and Practice. p. 349-377. 2017.

DUTTA, D. K; GWEBU, K. L; WANG, J. Personal innovativeness in technology, related knowledge and experience, and entrepreneurial intentions in emerging technology industries: a process of causation or effectuation?. International Entrepreneurship and Management Journal. v. 11, p. 529-555. 2015.

EVALD, M. R.; SENDEROVITZ, M. Exploring internal corporate venturing in SMEs: effectuation at work in a new context. Journal of Entreprising Culture. v. 21, n. 3, p. 275299. 2013.

EYANA, S. M; MASUREL, E.; PAAS, L. J. Causation and effectuation behaviour of Ethiopian entrepreneurs: Implications on performance of small tourism firms. Journal of 
Small Business and Enterprise Development. 2017. Disponível em: < https://doi.org/10.1108/JSBED-02-2017-0079>. Acesso em março de 2018.

FAIA, V. S.; ROSA, M. A. G.; MACHADO, H. P. V. Alerta empreendedor e as abordagens Causation e Effectuation sobre empreendedorismo. Revista de Administração Contemporânea, Rio de Janeiro, v. 18. n. 2, p. 196-216. 2014.

FUCHS, S.C.; PAIM, B. S. Revisão sistemática de estudos organizacionais com meta análise. Revista HPCA, Porto Alegre, v. 30, n. 3, p. 294-301. 2010.

FUTTERER, F.; SCHMIDT, J.; HEIDENREICH, S. Effectuation or causation as the key to corporate venture success? Investigating effects of entrepreneurial behaviors on business model innovation and venture performance. Long Range Planning. v. 51, p. 64-81. 2018.

GALVÃO, T.F ; PEREIRA, M.G. Revisões sistemáticas da literatura: passos para sua elaboração. Epidemiol. Serv. Saúde, Brasília, v. 23, n.1, p. 183-184, jan-mar. 2014.

GALKINA, T.; CHETTY, S. Effectuation and Networking of Internationalizing SMEs. Management International Review. v. 55, p. 647-676. 2015.

GALKINA, T.; LUNDGREN-HENRIKSSON, E. L.; Coopetition as na entrepreneurial process: Interplay of causation and effectuation. Industrial Marketing Management. v. 67, p. 158-173. 2017.

GUO, R.; CAI, L.; ZHANG, W. Effectuation and causation in new internet venture growth: The mediating effect of resource bundling strategy. Internet Research. v. 26, n. 2, p.460-483. 2016.

GÜNZEL-JENSEN, F.; ROBINSON, S. Effectuation in the undergraduate classroom: three barriers to entrepreneurial learning. Education + Training, v. 59, n. 7/8, p.780-796. 2017.

HELMERSON, H.; MATTSON, J. Text-analytic measurement of effectuation and causation orientations among small and global business managers. Qual Quant. v. 47, p. 3493-3507. 2013.

HES, T.; SULAIMAN, H.; BALI CHÁVEZ G.; MINTAH, S.; SALMAN, A. Comparison of four microfinance markets from the point of view of the effectuation theory, complemented by proposed musketeer principle illustrating forces within village banks. Management \& Marketing Challenges for the Knowledge Society. v. 12, n. 1, p. 37-48. 2017.

JISR, R. E.; MAAMARI, B. E. Effectuation: Exploring a Third Dimension to Tacit Knowledge. Research Article. v. 24, n. 1, p. 72-78. 2017.
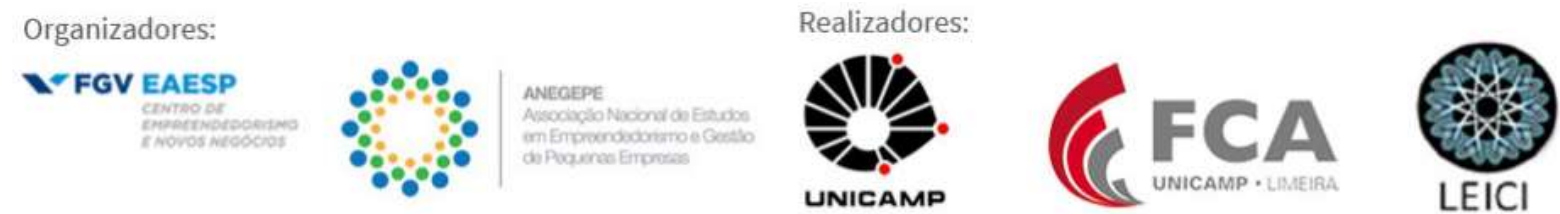
LAINE, I.; GALKINA, T. The interplay of effectuation and causation in decision making: Russian SMEs under institutional uncertainty. International Entrepreneurship and Management Journal, v. 13. p. 905-941. 2017.

LASKOVALA, A.; SHIROKOVA, G.; MORRIS, M. H. National culture, effectuation, and new venture performance: global evidence from student entrepreneurs. Small Businesses Economics. v. 49, p. 687-709. 2017.

LEUCZ, T.; ANDREASSI, T.; O processo decisório e o uso das lógicas effectuation e causation, frente à transição da pequena para a média empresa: casos do setor hoteleiro na cidade de Curitiba-PR. Revista Brasileira de Pesquisa em Turismo, São Paulo, v. 9, n. 3, p. 400-421. 2015.

LONG, D.; XIA Z., HU, W-B. How does entrepreneurial opportunity affect the decisionmaking process of effectuation?: Evidence from China. Kybernetes, v. 46, n. 6, p. 980-999. 2017.

MATALAMÄKI, M. J. Effectuation, an emerging theory of entrepreneurship - towards a mature stage of the development. Journal of Small Business and Enterprise Development. v. 24, n. 4, p.928-949. 2017.

MATALAMÄKI, M.; VUORINEN, T.; VARAMÄKI, E.; SORAMA, K. Business Growth in Established Companies; Roles of Effectuation and Causation. Journal of Enterprising Culture, v. 25, n 2, p123-148. 2017.

MATTA, J. R. N.; MELLO, R. D. C. A internacionalização de uma empresa brasileira de software de segurança à luz das teorias de redes e effectuation. Revista Eletrônica de Negócios Internacionais, São Paulo, v. 9, n. 3, p. 78-94. 2014.

MELINDA, T.; SUTANTO, J. E.; CHRISTIAN, S. Effective of effectuation-based Entrepreneurship Learning. Liceo Journal of Higher Education Research, v. 11, n.15, p. 107-118. 2015.

MELINDA, T.; SUTANTO, J. E.; CHRISTIAN, S. The development of effectuation-based learning model to increase the ability of University Students in Running a Business. Liceo Journal of Higher Education Research, v. 10, n. 1, p. 178-190. 2014.

MURDOCK, K. A; VARNES, C. J. Beyond effectuation: Analysing the transformation of business ideas into ventures using actor-network theory. International Journal of Entrepreneurial Behavior \& Research, v. 24, n.1, p. 256-272. 2018.

ORTEGA, A. M.; GARCÍA, M. T.; SANTOS, M. V. Effectuation-causation: what happens in new product development? Management Decision, v. 55, n. 8, p. 1717-1735. 2017. 
PARIDA, V.; GEORGE, N. M.; LAHTI, T.; WINCENT, J. Influence of subjetive interpretation, causation, and effectuation on initial venture sale. Journal of Business Research, v. 69. p. 4815-4819. 2016.

PELOGIO, E. A.; ROCHA, L. C. S.; MACHADO, H. V.; AÑEZ, M. E. M. Empreendedorismo e estratégia sob a ótica da lógica effectuation. Revista Ibero-Americana de Estratégia (RIAE), São Paulo, v. 12, n. 2, p. 228-249. 2013.

PELOGIO, E. A.; ROCHA, L. C. S. Modelo de decisão effectuation: uma alternativa para o estudo da criação de novos negócios. HOLOS, Natal, v. 8, p. 211-224. 2016.

PORTO, P.; MELLO, R. C. Empreendedorismo internacional e Effectuation: O caso do Café Yaguara Ecológico. Revista Eletrônica de Negócios Internacionais, São Paulo, v.10, n. 3, p. 15-30. 2015.

REYMEN, I.; BERENDS, H.; OUDEHAND, R.; STULTIËNS, R. Decision making for business model development: a process study of effectuation and causation in new technology-based ventures. R \& D Management, v. 47, n. 4, p. 595-606. 2016.

REYMEN, I. M. M. J.; ANDRIES, P.; BERENDS, H.; MAUER, R.; STEPHAN U.; BURG, E. V. Understanding dynamics of strategic decision making in venture creation: a process study of effectuation and causation. Strategic Entrepreneurship Journal,. v. 9, p. 351-379, 2015 .

ROACH, D. C.; RYMAN, J. A.; MAKANI, J. Effectuation, innovation and performance in SMEs: an empirical study. European Journal of Innovation Management. v.19, n. 2, p.214-238. 2016.

SALUSSE, M. A.Y.; ANDREASSI, T. O ensino de empreendedorismo com fundamento na Teoria Effectuation. RAC, Rio de Janeiro, v. 20, n. 3, p. 305-327. 2016.

SARASVATHY, S. Effectuation: Elements of Entrepreneurial Expertise. Cheltenham, UK: Edward Elgar, 2008.

SARMENTO, C. F. B.; CARVALHO, C. A. S.; DIB, L. A. R. Effectuation and the influence of social networks on the internationalization of accelerated startups. Revista Eetrônica de Negócios Internacionais, São Paulo, v. 11, n. 1, p. 63-73. 2016.

SCHMIDT, S.; BENDING, D.; BRETTEL, M. Building an equity story: the impact of effectuation on business angel investments. Journal of Business Economics. 2017. Disponível em : < https://doi.org/10.1007/s11573-017-0868-2>. Acesso em fevereiro de 2018.

\section{Organizadores:}

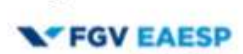

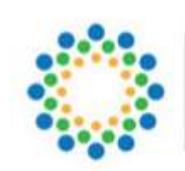

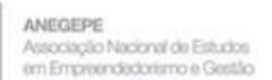

Realizadores:
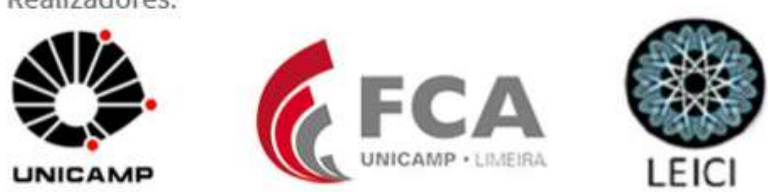
SILBERZAHN, P. Effectuation: Les principes de l'entrepreneurial pour tous. Pearson France, 2014.

SITOH, M. K.; PAN, S. L.; YU, C. Y. Business Models and Tactics in New Product Creation: The Interplay of Effectuation and Causation Processes. IEEE Transactions on Engineering Management, v. 61, n. 2. 2014.

STROE, S.; PARIDA, V.; WINCENT, J. Effectuation or causation: An fsQCA analysis of entrepreneurial passion, risk perception, and self-efficacy. Journal of Business Research. 2018. Disponível em < https://doi.org/10.1016/j.jbusres.2018.01.035> Acesso em fevereiro de 2018.

TRANFIELD, D.; DENYER, D.; SMART, P. Towards a methodology for developing evidence-informed man-agement knowledge by means of systematic review. British Journal of Management, London, v. 14, p. 207-222, 2003.

URBAN, B.; HEYDENRYCH, J. Technology orientation and effectuation - links to firm performance in the renewable energy sector of south africa. South African Journal of Industrial Engineering, v. 26, n. 3, p 125-136. 2015.

VILLANI, E.; LINDER, C.; GRIMALDI, R. Effectuation and causation in science-based new venture creation: a configurational approach. Journal of Business Research, v. 83, p. 173185. 2018.

WELTER, C.; KIM, S. Effectuation under risk and uncertainty: a simulation model. Journal of Business Venturing, v. 33, p. 100-116. 2018.

YU, X.; TAO, Y.; TAO, X.; XIA, F.; LI, Y. Managing uncertainty in emerging economies: The interaction effects between causation and effectuation on firm performance.

Technological Forecasting \& Social Change. 2017. Disponível em < https://doi.org/10.1016/j.techfore.2017.11.017>. Acesso em março de 2018. 